\title{
Time and charge calibration of the LHAASO electromagnetic particle detectors
}

\section{Binyu Pang, ${ }^{a, *}$ Hongkui Lv, ${ }^{b}$ Xiangdong Sheng, ${ }^{b}$ Jia Liu, ${ }^{b}$ Xiaopeng Zhang $^{b}$ and Yu $\mathrm{He}^{a}$ on behalf of the LHAASO Collaboration}

(a complete list of authors can be found at the end of the proceedings)

${ }^{a}$ School of Physical Science and Technology, Southwest Jiaotong University, Chendou 610031, China

${ }^{b}$ Institute of High Energy Physics, Chinese Academy of Sciences, Beijing 100049, China

E-mail: heyujy@swjtu.edu.cn

\begin{abstract}
:
The one square kilometer array (KM2A), a sub-array of Large High Altitude Air Shower Observatory (LHAASO) experiment, consists of 5195 electromagnetic particle detectors (EDs) and 1188 muon detectors (MDs), has been built over three-quarters scale. Its main scientific goal is to search gamma-ray sources at energies above $100 \mathrm{TeV}$. Offline calibration method has been used to calibrate thousands of EDs to guarantee the key performances of the array such as angular resolution and pointing accuracy within $0.1^{\circ}$ during long-term operation. The experimental results of the $3 / 4$ array show that this method can be used to determine the detector time offset with an accuracy of $0.5 \mathrm{~ns}$ and the particle number density with an accuracy of a few percent. Furthermore, we monitor the calibration parameters during the array operation and update the calibration results regularly to ensure the data quality of the detector.
\end{abstract}

$37^{\text {th }}$ International Cosmic Ray Conference (ICRC 2021)

July 12th - 23rd, 2021

Online - Berlin, Germany

\footnotetext{
*Presenter
} 


\section{Introduction}

LHAASO is located on Haizi Mountain in Daocheng, Sichuan Province, China. It is a large hybrid extensive air shower (EAS) array consists of three sub-arrays (Fig. 1), namely a $1.3 \mathrm{~km}^{2}$ array (KM2A), water Cherenkov detector array (WCDA) and wide field-of-view air Cherenkov/fluorescence telescopes array (WFCTA) [1]. KM2A is unique for its unprecedented sensitivity for gamma-ray sources at energies above $20 \mathrm{TeV}$. $5195 \mathrm{EDs}$ in KM2A are deployed to detect densities and arrival time of EAS charged particles produced by the primary particles, from which the primary energy and direction can be reconstructed. In order to guarantee the angular resolution of the array better than $0.5^{\circ}$ above $30 \mathrm{TeV}$ and the pointing accuracy better than $0.1^{\circ}$, the time synchronization accuracy of EDs must to be within $1 \mathrm{~ns}$. Besides, primary energy reconstruction depends on the measured signal integrated charge recorded by each fired ED units, a reliable detector charge calibration is required for energy interpretation [2].

Half of the KM2A array has been operated since December 2019 and the three-quarters has been operated since December 2020. Until now, about 3900 EDs and 890 MDs have been installed and running. An offline calibration method which relies on the measurement of charged particles within the EASs was initially applied in half scale array and extended to 3/4 array. Simultaneously, we monitor the calibration parameters for several months to ensure the stability of the array operation and physical results. This study also focus on the applicability to the full array that will be built.

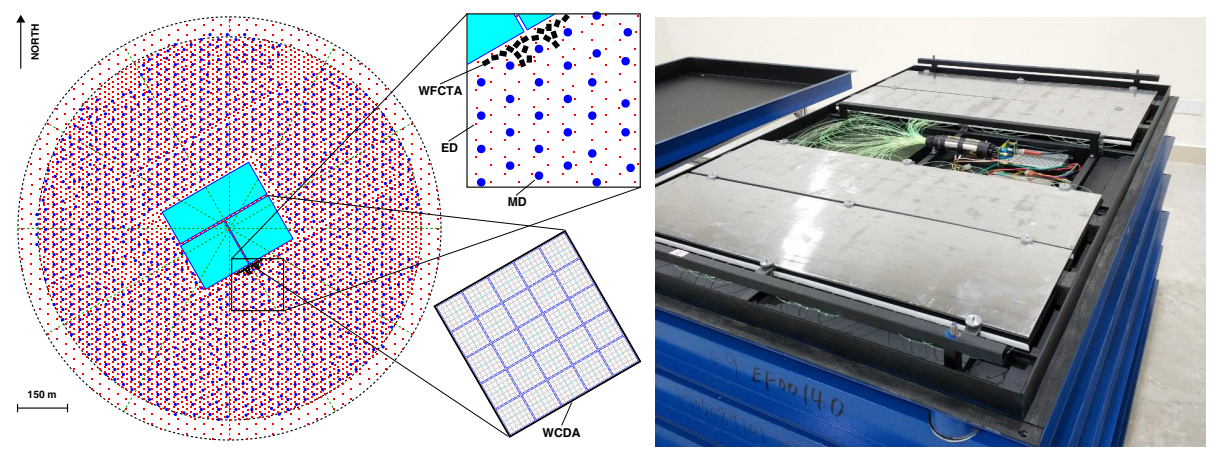

Figure 1: Left: The layout of the LHAASO experiment. LHAASO consists of one square kilometer array (KM2A), water Cherenkov detector array (WCDA) and wide-field Cherenkov telescope array (WFCTA). Right: Schematic of a ED.

\section{Time calibration}

The ED consists of four plastic scintillation tiles of $100 \mathrm{~cm} \times 25 \mathrm{~cm} \times 1 \mathrm{~cm}$ each, several wavelength-shifting fibers, one 1.5 inch photomultiplier tube (PMT), a front-end electronics (FEE) (Fig. 1). The charged particles hit the plastic scintillator and produce photons. The photons are transmitted to the photocathode of PMT through the wavelength shifting fibers and converted into electronic pulses, finally, the signal is recorded by FEE [3]. The time offset is measured as a cumulative effect of several processes described above. The main uncertainties on the measured hit times come from the time offset spread among the EDs [4]. A reliable reconstruction of the primary gamma-ray direction requires the accurate determination of the arrival time of EAS particles on 
each detector. Therefore, the time synchronization of thousands of EDs is crucial to guarantee the performance of the array to achieve the expected.

\subsection{Time calibration principle}

The offline calibration is an automatic self-calibration method, which uses EAS charged particles as the calibration beam. Since the EAS front approximately sustains a conical shape (Fig. 2). The time offset of the i-th ED $\Delta t_{i}$ located at position coordinates $\left(x_{i}, y_{i}\right)$ is determined, event by event, as follows:

$$
\left.\Delta t_{i}=t_{i}-t_{i}^{r e a l}=\left[(l-\bar{l}) \frac{x_{i}}{c}+(m-\bar{m}) \frac{y_{i}}{c}\right)+\sqrt{1-(l-\bar{l})^{2}-(m-\bar{m})^{2}} \frac{z_{i}}{c}+\alpha r_{i}+t_{0}\right]
$$

where $t_{i}$ and $t_{i}^{r e a l}$ are the measured arrival time of EAS particle for i-th ED and the expected "real" one, respectively; $l$ and $m$ are two components of the reconstructed direction vector ( $l=\sin \theta \cos \phi$, $m=\sin \theta \sin \phi(\theta$ and $\phi$ are the zenith and azimuth angles, respectively $)) ;$
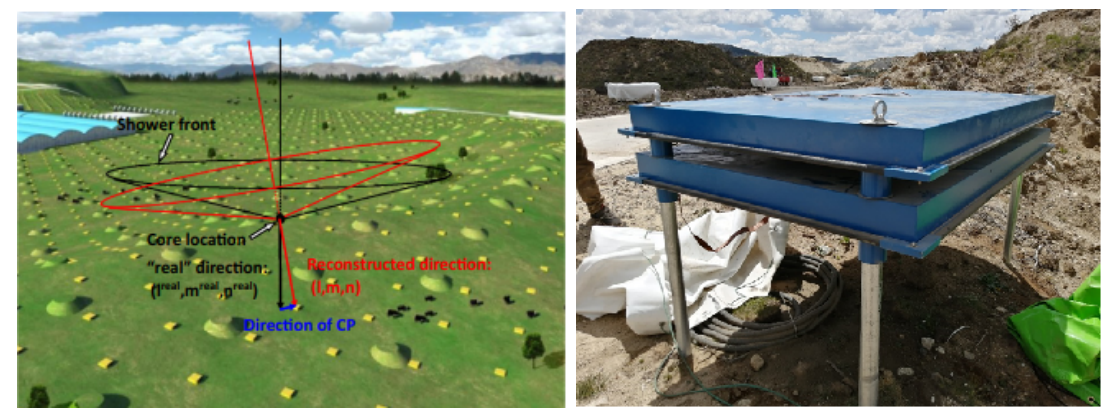

Figure 2: Left: Schematic of a shower front and the CP introduced by detector time offset; Right: A muon telescope system consists of two EDs.

To get the accurate direction of EAS, the two directions $(l, m)$ have been modified by using the mean value of reconstructed direction $(l, m)$, which is the so-called characteristic plane (CP) described in [5]; Other parameters in the equation such as the conicity coefficient $\alpha$ and the time parameter $t_{0}$ are obtained from the fitting process of direction reconstruction, and the transverse distance of the i-th ED from the shower core $r_{i}$ can be obtained as the shower core is reconstructed.

\subsection{Time calibration results}

We selected 9 hours of data and determined the time offset by fitting the most probable value of the time residuals distribution which are calculated according to Equation 1. As shown in Figure 3, the time offset distribution width of 3937 EDs in the 3/4 array is less than $1 \mathrm{~ns}$.

To verify the applicability of this method and estimate its precision, hardware calibration is performed using a muon telescope system. The muon system consists of two EDs was established in the KM2A, as show in Figure 2. The top layer is the probe detector and bottom layer is the detector to be calibrated. Once background muons pass through the probe detector and the detector under calibration simultaneously, the differencesof the time offset between these two detectors can be determined. The relative time offsets are then measured using a probe detector moved above all 

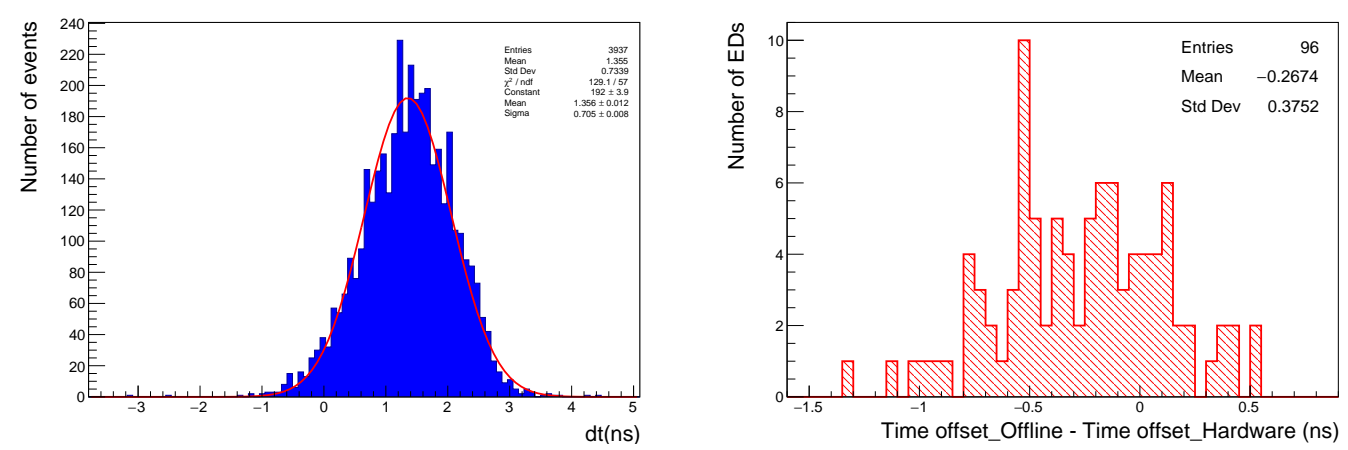

Figure 3: Left: The time offsets distribution of EDs in the 3/4 array; Right: The differences distribution between hardware calibration and offline calibration.

the detector units as reference. This calibration method can achieve a high precision of $0.1 \mathrm{~ns}$ under suitable statistics. Using the muon telescope system, the relative time offset of more than 90 EDs sampled from the KM2A are calibrated.

Comparing of the ED time offset results determined by two independent methods yields a direct prediction of the precision of the calibration method. As shown in Figure 3, the root mean square of the differences between ED time offsets obtained from two calibration methods is $0.4 \mathrm{~ns}$, which is well within the required precision of the experiment.

\section{Charge calibration}

The energy reconstruction depends on PMT signal integrated charge recorded by each fired EDs. Converting the integrated charge from ADC units to particle number is crucial for the accuracy of energy reconstruction to provide a common reference standard for energy reconstruction between individual EDs. The charged particles within the shower front are used as the calibration particle beam.The most probable value (MPV) of integrated charge spectrum is fitted to get the amplitude of charge corresponding to a single particle signal, then the charge calibration is performed using the MPV value above.

The lateral distribution of charged particle density within the EAS is simulated using the CORSIKA software. According to the lateral development, the density of particles decreases as the radial distance from the shower core increases (Fig. 4). Therefore, a more rigorous single MIP spectrum can be obtained by selecting the ED signal at the large radial distance from the shower core (Fig. 5). The black line in Figure 5 indicates that there is the integrated charge spectrum without any cut of distance. The blue line is the integrated charge spectrum selected from the ED single at the distance greater than $100 \mathrm{~m}$, the purity of the single particle signal is greater than $95 \%$ as the number of particles arrived at each ED follows a Poisson distribution. The light blue line is the integrated charge spectrum selected from the ED single at the distance greater than $200 \mathrm{~m}$. After data selection, most of the multiparticle components are removed by comparing with the tails of these spectrums, however the peak value is almost unchanged (although their MPV value has a little shift within 5\%). The MPV of the charge distribution of 3964 EDs in 3/4 array is shown in Figure 5. 


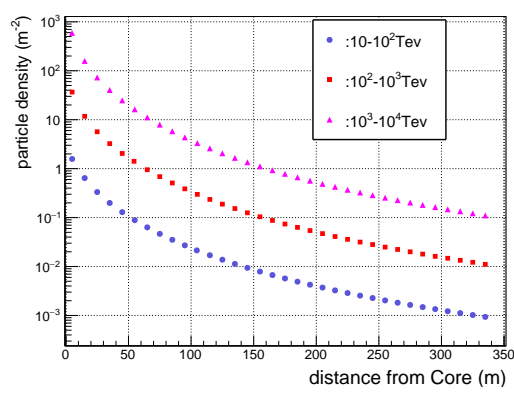

Figure 4: Average lateral distribution for electrons and positrons in showers initiated by vertical protons
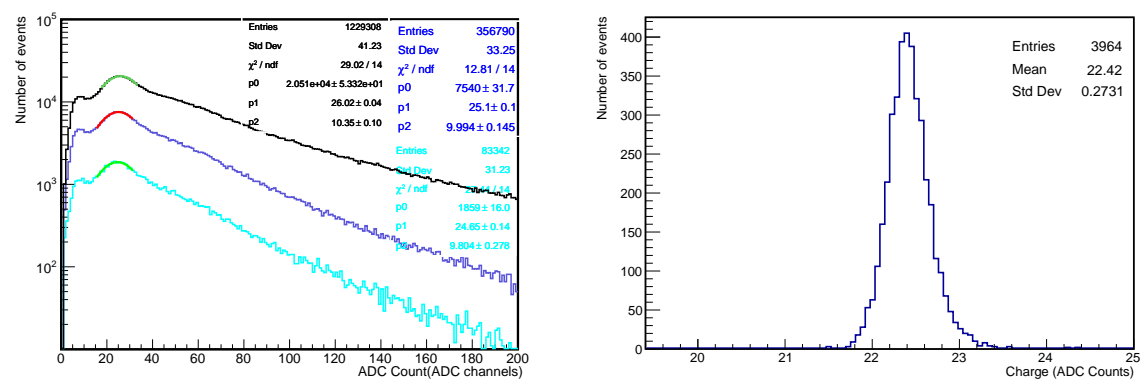

Figure 5: Left: Single MIP spectrum is obtained by the offline calibration method (black line). The blue line is the data selected from the distance greater than $100 \mathrm{~m}$. The data at the distance greater than $200 \mathrm{~m}$ is selected (light blue line); Right: The MPV distribution of EDs in 3/4 array.

\section{Calibration parameters monitoring}

Long-term monitoring of the calibration parameters is helpful to understand the performance
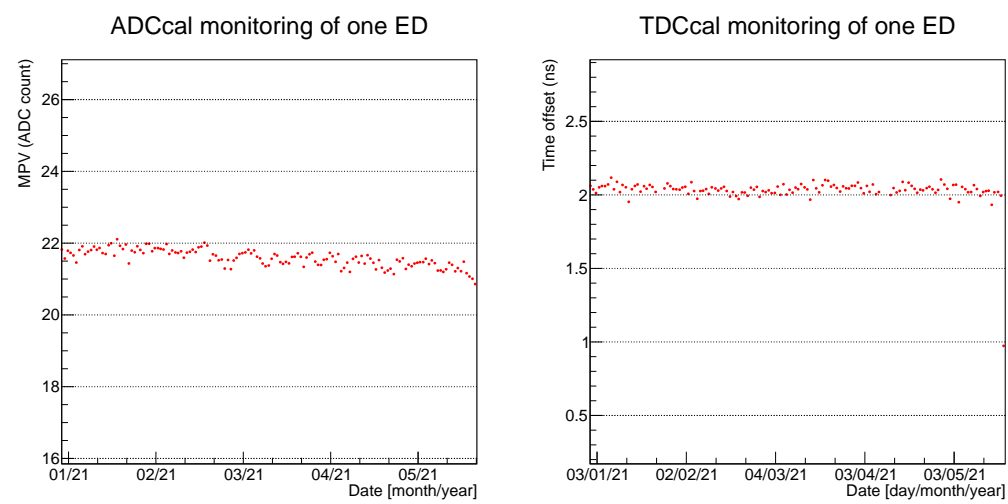

Figure 6: Left: Monitoring of charge calibration parameter of a single ED, the MPV is affected by temperature; Right: Long-term stability distribution of the time offset of a single ED.

of the detector and array stability. 1/2 array has been operating steadily more than one year and 3/4 array has been operating for half year. This allows us to analyze the long-term stability of the calibration parameters. The MPV of integrated charge distribution and the time offset value for each ED have been monitored (Fig. 6). The time offset values have a little fluctuation, it also reflects 
that the detector is very stable. The calibration file is updated periodically to ensure the accuracy of event reconstruction and stability of the array performance. In addition, the offline calibration also offers an ideal method to judge the working state of the detector.

\section{Conclusion}

The construction of LHAASO experiment will be completed this year, and there will be more than 5000 detectors. Nowadays, the offline calibration has been successfully applied to 3/4 array. The relative time offsets can be periodically calibrated with a precision of better than $1 \mathrm{~ns}$ and charge calibration precision is sufficient within the KM2A performance requirements. Furthermore, we set up a monitoring program to monitor the calibration parameters in real time. This allows us to deal with the anomaly detector as quickly as possible. The calibration method is not only applicable to LHAASO, but also has reference significance for a new generation of EAS experiments comprising huge number detectors covering a large area.

\section{Acknowledgements}

This work is supported in China by National Key R\&D program of China (grant number 2018YFA0404201), It is also supported by the Fundamental Research Funds for the Central Universities (grant numbers 2682020CX77, 2682020CX73, 2682020CX74) and by the Science and Technology Department of Sichuan Province (grant numbers 2021YFSY0031, 2020YFSY0016), and by NSFC (grant number 11947404).

\section{References}

[1] S.Z.Chen, et al., Chinese Physics C, 2021, 45 (02): 522-534

[2] Hongkui Lv, et al., Astroparticle Physics 100 (2018) 22-28.

[3] Hongkui Lv, Y.C.Nan, et al., proceeding of 36th ICRC, (2019)

[4] Hongkui Lv, et al., proceeding of 35th ICRC, (2017)

[5] H.H.He, et al., Astroparticle Physics 27 (2007), 528-532. 


\section{Full Authors List: LHAASO Collaboration}

Zhen $\mathrm{Cao}^{1,2,3}$, F. Aharonian ${ }^{4,5}$, Q. An ${ }^{6,7}$, Axikegu ${ }^{8}$, L.X. Bai ${ }^{9}$, Y.X. Bai ${ }^{1,3}$, L.X. Bai ${ }^{9}$, Y.X. Bai ${ }^{1,3}$, Y.W. Bao ${ }^{10}$, D. Bastieri ${ }^{11}$, X.J. Bi ${ }^{1,2,3}$, Y.J. Bi ${ }^{1,3}$, H. Cai ${ }^{12}$, J.T. Cai ${ }^{11}$, Zhe Cao ${ }^{6,7}$, J. Chang ${ }^{13}$, J.F. Chang ${ }^{1,3,6}$, B.M. Chen ${ }^{14}$, E.S. Chen ${ }^{1,2,3}$, J. Chen ${ }^{9}$, Liang Chen $^{1,2,3}$, Liang Chen ${ }^{15}$, Long Chen ${ }^{8}$, M.J. Chen ${ }^{1,3}$, M.L. Chen ${ }^{1,3,6}$, Q.H. Chen ${ }^{8}$, S.H. Chen ${ }^{1,2,3}$, S.Z. Chen ${ }^{1,3}$, T.L. Chen ${ }^{16}$,X.L. $\mathrm{Chen}^{1,2,3}$, Y. Chen $^{10}$, N. Cheng ${ }^{1,3}$, Y.D. Cheng ${ }^{1,3}$, S.W. Cui ${ }^{14}$, X.H. Cuii ${ }^{17}$, Y.D. Cui ${ }^{18}$, B. D'Ettorre Piazzoli ${ }^{19}$, B.Z. Dai ${ }^{20}$, H.L. Dai $^{1,3,6}$, Z.G. Dai ${ }^{7}$, Danzengluobu ${ }^{16}$, D. della Volpe ${ }^{21}$, X.J. Dong ${ }^{1,3}$, K.K. Duan ${ }^{13}$, J.H. Fan ${ }^{11}$, Y.Z. Fan ${ }^{13}$, Z.X. Fan ${ }^{1,3}$, J. Fang ${ }^{20}$, K. Fang $^{1,3}$, C.F. Feng ${ }^{22}$, L. Feng ${ }^{13}$, S.H. Feng ${ }^{1,3}$, Y.L. Feng ${ }^{13}$, B. Gao ${ }^{1,3}$, C.D. Gao ${ }^{22}$, L.Q. Gao ${ }^{1,2,3}$, Q. Gao ${ }^{16}$, W. Gao ${ }^{22}$, M.M. Ge $^{20}$, L.S. Geng ${ }^{1,3}$, G.H. Gong ${ }^{23}$, Q.B. Gou ${ }^{1,3}$, M.H. Gu ${ }^{1,3,6}$, F.L. Guo ${ }^{15}$, J.G. Guo ${ }^{1,2,3}$, X.L. Guo ${ }^{8}$, Y.Q. Guo ${ }^{1,3}$, Y.Y. Guo ${ }^{1,2,3,13}$, Y.A. $\mathrm{Han}^{24}$, H.H. He $\mathrm{H}^{1,2,3}$, H.N. He ${ }^{13}$, J.C. He ${ }^{1,2,3}$, S.L. He ${ }^{11}$, X.B. He ${ }^{18}$, Y. He ${ }^{8}$, M. Heller ${ }^{21}$, Y.K. Hor ${ }^{18}$, C. Hou ${ }^{1,3}$, H.B. Hu ${ }^{1,2,3}$, S.

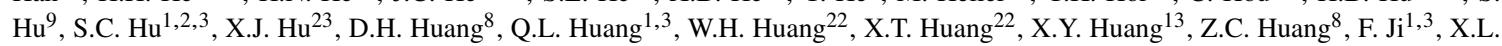
$\mathrm{Ji}^{1,3,6}$, H.Y. Jia ${ }^{8}$, K. Jiang ${ }^{6,7}$, Z.J. Jiang ${ }^{20}$, C. Jin ${ }^{1,2,3}$, T. Ke ${ }^{1,3}$, D. Kuleshov ${ }^{25}$, K. Levochkin ${ }^{25}$, B.B. Li ${ }^{14}$, Cheng Li ${ }^{6,7}$, Cong Li ${ }^{1,3}$,

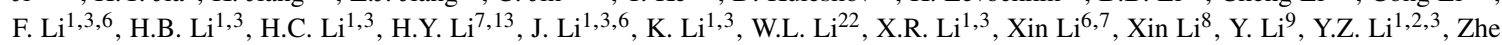

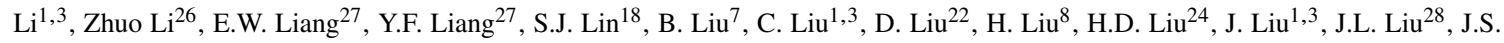

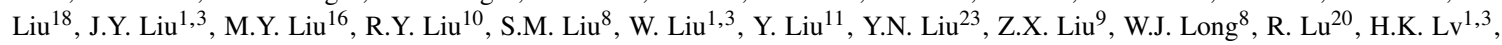

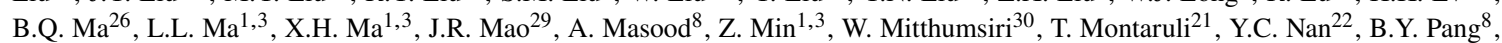
P. Pattarakijwanich ${ }^{30}$, Z.Y. Pei ${ }^{11}$, M.Y. Qi ${ }^{1,3}$, Y.Q. Qi ${ }^{14}$, B.Q. Qiao ${ }^{1,3}$, J.J. Qin ${ }^{7}$, D. Ruffolo ${ }^{30}$, V. Rulev ${ }^{25}$, A. Sáiz ${ }^{30}$, L. Shao ${ }^{14}$, O. Shchegolev $^{25,31}$, X.D. Sheng ${ }^{1,3}$, J.Y. Shi ${ }^{1,3}$, H.C. Song ${ }^{26}$, Yu.V. Stenkin ${ }^{25,31}$, V. Stepanov ${ }^{25}$, Y. Su ${ }^{32}$, Q.N. Sun ${ }^{8}$, X.N. Sun ${ }^{27}$, Z.B.

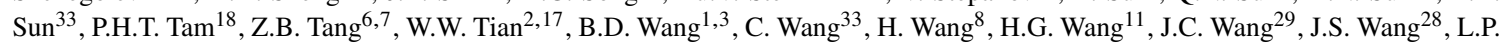
Wang $^{22}$, L.Y. Wang ${ }^{1,3}$, R.N. Wang ${ }^{8}$, W. Wang ${ }^{18}$, W. Wang ${ }^{12}$, X.G. Wang ${ }^{27}$, X.J. Wang ${ }^{1,3}$, X.Y. Wang ${ }^{10}$, Y. Wang ${ }^{8}$, Y.D. Wang ${ }^{1,3}$, Y.J. Wang $^{1,3}$, Y.P. Wang ${ }^{1,2,3}$, Z.H. Wang ${ }^{9}$, Z.X. Wang ${ }^{20}$, Zhen Wang ${ }^{28}$, Zheng Wang ${ }^{1,3,6}$, D.M. Wei $^{13}$, J.J. Wei ${ }^{13}$, Y.J. Wei ${ }^{1,2,3}$, T. Wen ${ }^{20}$, C.Y. $\mathrm{Wu}^{1,3}$, H.R. $\mathrm{Wu}^{1,3}$, S. Wu ${ }^{1,3}$, W.X. Wu ${ }^{8}$, X.F. Wu ${ }^{13}$, S.Q. Xi ${ }^{1,3}$, J. Xia ${ }^{7}, 13$, J.J. Xia ${ }^{8}$, G.M. Xiang ${ }^{2,15}$, D.X. Xiao ${ }^{16}$, G. Xiao ${ }^{1,3}$, H.B. Xiao ${ }^{11}$, G.G. Xin ${ }^{12}$, Y.L. Xin ${ }^{8}$, Y. Xing ${ }^{15}$, D.L. Xu ${ }^{28}$, R.X. Xu ${ }^{26}$, L. Xue ${ }^{22}$, D.H. Yan ${ }^{29}$, J.Z. Yan ${ }^{13}$, C.W. Yang 9 , F.F. Yang ${ }^{1,3,6}$, J.Y. Yang ${ }^{18}$, L.L. Yang ${ }^{18}$, M.J. Yang ${ }^{1,3}$, R.Z. Yang ${ }^{7}$, S.B. Yang ${ }^{20}$, Y.H. Yao ${ }^{9}$, Z.G. Yao ${ }^{1,3}$, Y.M. Ye ${ }^{23}$, L.Q. Yin ${ }^{1,3}$, N. Yin ${ }^{22}$, X.H. You $^{1,3}$, Z.Y. You ${ }^{1,2,3}$, Y.H. Yu ${ }^{22}$, Q. Yuan ${ }^{13}$, H.D. Zeng ${ }^{13}$, T.X. Zeng ${ }^{1,3,6}$, W. Zeng ${ }^{20}$, Z.K. Zeng ${ }^{1,2,3}$, M. Zha ${ }^{1,3}$, X.X. Zhai ${ }^{1,3}$, B.B. Zhang $^{10}$, H.M. Zhang ${ }^{10}$, H.Y. Zhang ${ }^{22}$, J.L. Zhang ${ }^{17}$, J.W. Zhang ${ }^{9}$, L.X. Zhang ${ }^{11}$, Li Zhang ${ }^{20}$, Lu Zhang ${ }^{14}$, P.F. Zhang ${ }^{20}$, P.P. Zhang ${ }^{14}$, R. Zhang ${ }^{7,13}$, S.R. Zhang ${ }^{14}$, S.S. Zhang ${ }^{1,3}$, X. Zhang ${ }^{10}$, X.P. Zhang ${ }^{1,3}$, Y.F. Zhang ${ }^{8}$, Y.L. Zhang ${ }^{1,3}$, Yi Zhang ${ }^{1,13}$, Yong Zhang ${ }^{1,3}$, B. $Z_{\text {Zhao }}^{8}$, J. Zhao ${ }^{1,3}$, L. Zhao ${ }^{6,7}$, L.Z. Zhao ${ }^{14}$, S.P. Zhao ${ }^{13,22}$, F. Zheng ${ }^{33}$, Y. Zheng ${ }^{8}$, B. Zhou ${ }^{1,3}$, H. Zhou ${ }^{28}$, J.N. Zhou ${ }^{15}$, P. Zhou ${ }^{10}$, R. Zhou $^{9}$, X.X. Zhou ${ }^{8}$, C.G. Zhu ${ }^{22}$, F.R. Zhu ${ }^{8}$, H. Zhu ${ }^{17}$, K.J. Zhu ${ }^{1,2,3,6}$ and X. Zuo ${ }^{1,3}$

${ }^{1}$ Key Laboratory of Particle Astrophyics \& Experimental Physics Division \& Computing Center, Institute of High Energy Physics, Chinese Academy of Sciences, 100049 Beijing, China.

${ }^{2}$ University of Chinese Academy of Sciences, 100049 Beijing, China.

${ }^{3}$ TIANFU Cosmic Ray Research Center, Chengdu, Sichuan, China.

${ }^{4}$ Dublin Institute for Advanced Studies, 31 Fitzwilliam Place, 2 Dublin, Ireland.

${ }^{5}$ Max-Planck-Institut for Nuclear Physics, P.O. Box 103980, 69029 Heidelberg, Germany.

${ }^{6}$ State Key Laboratory of Particle Detection and Electronics, China.

${ }^{7}$ University of Science and Technology of China, 230026 Hefei, Anhui, China.

${ }^{8}$ School of Physical Science and Technology \& School of Information Science and Technology, Southwest Jiaotong University, 610031 Chengdu, Sichuan, China.

${ }^{9}$ College of Physics, Sichuan University, 610065 Chengdu, Sichuan, China.

${ }^{10}$ School of Astronomy and Space Science, Nanjing University, 210023 Nanjing, Jiangsu, China.

${ }^{11}$ Center for Astrophysics, Guangzhou University, 510006 Guangzhou, Guangdong, China.

${ }^{12}$ School of Physics and Technology, Wuhan University, 430072 Wuhan, Hubei, China.

${ }^{13}$ Key Laboratory of Dark Matter and Space Astronomy, Purple Mountain Observatory, Chinese Academy of Sciences, 210023 Nanjing, Jiangsu, China.

${ }^{14}$ Hebei Normal University, 050024 Shijiazhuang, Hebei, China.

${ }^{15}$ Key Laboratory for Research in Galaxies and Cosmology, Shanghai Astronomical Observatory, Chinese Academy of Sciences, 200030 Shanghai, China.

${ }^{16}$ Key Laboratory of Cosmic Rays (Tibet University), Ministry of Education, 850000 Lhasa, Tibet, China.

${ }^{17}$ National Astronomical Observatories, Chinese Academy of Sciences, 100101 Beijing, China.

${ }^{18}$ School of Physics and Astronomy \& School of Physics (Guangzhou), Sun Yat-sen University, 519000 Zhuhai, Guangdong, China.

${ }^{19}$ Dipartimento di Fisica dell'Università di Napoli 'Federico II', Complesso Universitario di Monte Sant’Angelo, via Cinthia, 80126 Napoli, Italy.

${ }^{20}$ School of Physics and Astronomy, Yunnan University, 650091 Kunming, Yunnan, China.

${ }^{21}$ D'epartement de Physique Nucl'eaire et Corpusculaire, Facult'e de Sciences, Universit'e de Gen 'eve, 24 Quai Ernest Ansermet, 1211 Geneva, Switzerland.

${ }^{22}$ Institute of Frontier and Interdisciplinary Science, Shandong University, 266237 Qingdao, Shandong, China.

${ }^{23}$ Department of Engineering Physics, Tsinghua University, 100084 Beijing, China.

${ }^{24}$ School of Physics and Microelectronics, Zhengzhou University, 450001 Zhengzhou, Henan, China.

${ }^{25}$ Institute for Nuclear Research of Russian Academy of Sciences, 117312 Moscow, Russia. 
${ }^{26}$ School of Physics, Peking University, 100871 Beijing, China.

${ }^{27}$ School of Physical Science and Technology, Guangxi University, 530004 Nanning, Guangxi, China.

${ }^{28}$ Tsung-Dao Lee Institute \& School of Physics and Astronomy, Shanghai Jiao Tong University, 200240 Shanghai, China.

${ }^{29}$ Yunnan Observatories, Chinese Academy of Sciences, 650216 Kunming, Yunnan, China.

${ }^{30}$ Department of Physics, Faculty of Science, Mahidol University, 10400 Bangkok, Thailand.

${ }^{31}$ Moscow Institute of Physics and Technology, 141700 Moscow, Russia.

${ }^{32}$ Key Laboratory of Radio Astronomy, Purple Mountain Observatory, Chinese Academy of Sciences, 210023 Nanjing, Jiangsu, China.

${ }^{33}$ National Space Science Center, Chinese Academy of Sciences, 100190 Beijing, China. 